\title{
OS CONTORNOS DA SEGURANÇA ALIMENTAR NO ÂMBITO DO SISTEMA JURÍDICO MULTILATERAL DE COMÉRCIO
}

\author{
THE SHAPE OF FOOD SECURITY UNDER THE MULTILATERAL TRADING LEGAL \\ SYSTEM
}

Mariagrazia Alabrese ${ }^{1}$

\begin{abstract}
Resumo: Apesar do grande esforço da comunidade internacional, a falta de segurança alimentar persiste e piorou nos últimos anos. Entre os múltiplos elementos que podem afetar a segurança alimentar, o comércio na agricultura desempenha um papel significativo em termos positivos e negativos. Considerando a segurança alimentar no sistema multilateral de comércio, uma das questões mais importantes é o próprio conceito de segurança alimentar adotado pelas partes negociadoras. Esse conceito corresponde à definição mais utilizada em nível internacional, ou seja, a definição da FAO? O fórum da Organização Mundial do Comércio apresentou uma noção clara e consistente de segurança alimentar? Este artigo discute o conceito de segurança alimentar proveniente do sistema legal da OMC, examinando as regras do Acordo sobre Agricultura relevante e levando em conta o atual processo de reforma.
\end{abstract}

Palavras-chave: Segurança alimentar; Acordo da OMC sobre Agricultura; regras excepcionais; Direito à alimentação.

\begin{abstract}
Despite great effort by the international community, a lack of food security persists and has even worsened in the last few years. Amongst the multiple elements that can affect food security, trade in agriculture plays a significant role both in positive and negative terms. Considering food security under the multilateral trading system, one of the most important issues is the very concept of food security as adopted by the negotiating parties. Does it match the most commonly used definition at an international level, i.e. the FAO's definition? Has the World Trade Organization forum come up with a clear and consistent notion of food security? This article discusses the concept of food security emerging from the WTO legal system, examining the rules of the relevant Agreement on Agriculture and taking into account the current reform process.
\end{abstract}

Keywords: Food security; WTO Agreement on Agriculture; Exceptional rules; Right to food.

\section{INTRODUÇÃO}

De acordo com a última edição do "Global Report on Food Crises" ${ }^{2}$, cerca de 124 milhões de pessoas em 51 países enfrentaram insegurança alimentar aguda em 2017: 11 milhões de pessoas a mais em relação ao ano anterior (FSIN, 2018). Infelizmente, esses números estão alinhados com a edição de 2017 do documento intitulado "Estado da Segurança Alimentar e Nutrição no Mundo" (FAO, 2017), que destaca como em 2016 o número de pessoas cronicamente subnutridas no mundo cresceu e alcançou cerca de 815 milhões de pessoas, número em muito superior ao ano anterior, de 777 milhões. Este cenário assustador está se desenvolvendo na direção oposta à trajetória prevista pela "Agenda 2030 para o Desenvolvimento Sustentável" (ONU, 2015) e a "Década de Ação da ONU sobre Nutrição 2016-2025" (ONU, 2016), que apela a todos os países e partes interessadas a agir em conjunto para eliminar a fome e prevenir todas as formas de desnutrição até 2030. Assim, apesar da profusão de iniciativas por parte da comunidade internacional, a falta de segurança alimentar persiste e está, na verdade, se agravando. 
O comércio agrícola internacional desempenha um papel fundamental tanto no fortalecimento como na obstrução do status de segurança alimentar nos níveis global, nacional e individual. O presente artigo tem como objetivo compreender como o sistema jurídico multilateral considera a segurança alimentar e a maneira como a segurança alimentar é efetivamente moldada sob as regras do comércio internacional.

\section{RELAÇÕES ENTRE COMÉRCIO NA AGRICULTURA E SEGURANÇA ALI- MENTAR: UMA PERSPECTIVA ECONÔMICA E EMPÍRICA}

A definição mais comum de segurança alimentar é aquela construída pela FAO: "a segurança alimentar existe quando todas as pessoas têm acesso físico, social e econômico a alimentos suficientes, seguros e nutritivos para atender suas necessidades alimentares e preferências alimentares, proporcionando uma vida ativa e saudável. Os quatro pilares da segurança alimentar são disponibilidade, acesso, utilização e estabilidade. A dimensão nutricional é parte integrante do conceito de segurança alimentar" (FAO, 2009) ${ }^{3}$.

Cada um desses quatro pilares ${ }^{4}$ é intensamente influenciado pelo comércio de produtos agrícolas (MATTHEWS, 2014; CLAPP, 2015).

O primeiro deles, disponibilidade, refere-se à quantidade de alimentos disponível em um país ou área por meio de todas as formas de produção doméstica, importações, estoques de alimentos e ajuda alimentar. O comércio pode afetar a disponibilidade pois a importação de alimentos pode elevar a quantidade de alimentos disponíveis internamente naqueles países que não são autossuficientes. Por outro lado, as restrições e proibições à exportação podem pôr em perigo os países que sofrem de escassez de alimentos e que mais precisam adquirir commodities agrícolas do exterior.

Em relação ao acesso, três dimensões são levadas em consideração. O acesso físico é uma dimensão logística que requer que o alimento esteja disponível onde as pessoas possam realmente alcançá-lo. O acesso econômico implica que as pessoas tenham a capacidade financeira de adquirir regularmente alimentos adequados. $\mathrm{O}$ acesso sociocultural refere-se às barreiras sociais que limitam o acesso a alimentos a alguns grupos devido a razões sociais ou de gênero. O comércio impacta principalmente o acesso econômico, pois pode impulsionar o crescimento econômico e promover o acesso à alimentação. Além disso, o mercado aberto pode levar a preços baixos (mais acessíveis para os consumidores). Entretanto, os pequenos agricultores podem sofrer com os baixos preços dos alimentos, acabando por excluí-los dos círculos internacionais se não forem competitivos ou não puderem cumprir com os altos padrões exigidos pelas cadeias globais de fornecimento de alimentos.

O pilar da utilização refere-se à qualidade e os nutrientes dos alimentos consumidos, não apenas sua quantidade. Vários elementos afetam essa questão, como a seleção dos produtos alimentícios, sua conservação e preparação, bem como a absorção de nutrientes (indicando que um corpo está em boa saúde). A utilização de alimentos está, portanto, relacionada à água limpa, saneamento e assistência à saúde. Essa dimensão, na verdade, não se refere apenas à nutrição, mas também a outros elementos relacionados ao uso, conservação, processamento e preparação dos alimentos, de modo a ressaltar os problemas envolvidos na segurança de alimentos. A ligação entre o comércio e 
a utilização está na evidência de que o comércio agrícola afeta os regimes alimentares e as preferências alimentares (por exemplo, em termos da qualidade nutricional dos alimentos disponíveis). Isso pode ter um efeito positivo contra a desnutrição, mas também pode favorecer escolhas alimentares pouco saudáveis (tornando as comidas processadas mais baratas do que os alimentos mais saudáveis). Da mesma forma, há potenciais efeitos negativos em relação às dietas tradicionais, uma vez que os alimentos importados podem ser mais baratos e substituir os alimentos tradicionais e típicos de uma área geográfica. Este fenômeno pode afetar as preferências alimentares das pessoas, que estão relacionadas aos alimentos tradicionais e culturalmente aceitáveis ("segurança alimentar existe quando todas as pessoas, em todos os momentos, têm... acesso a... comida para satisfazer as suas... preferências alimentares" (FAO, 2009)).

Finalmente, a estabilidade está relacionada à necessidade de que os três pilares acima mencionados sejam estáveis ao longo do tempo e que não sejam afetados negativamente por fatores naturais, sociais, econômicos ou políticos. Como a estabilidade está ligada aos outros pilares, a forma como ela é afetada pelo comércio, por sua vez, está estritamente relacionada aos efeitos do comércio em cada pilar.

\section{COMO AS REGRAS MULTILATERAIS DE COMÉRCIO TRATAM A SEGU- RANÇA ALIMENTAR}

O tema da segurança alimentar no âmbito do regulamento da OMC precisa ser discutido nos termos de um dos acordos do pacote mais amplo de tratados da OMC em vigor desde 1995: o Acordo sobre Agricultura ${ }^{5}$. Isso não inclui uma definição de segurança alimentar, uma vez que, como observado, "a segurança alimentar não é uma questão para as regras em si, mas é algo separado [...]"6 (SMITH, 2012). Apesar disso, o Acordo dedica ampla atenção à segurança alimentar. Isso se dá provavelmente em decorrência de um dos valores que esteve entre as forças motrizes da Rodada Uruguai de negociações e que resultou em um regime especial para a agricultura: os Estados não aceitaram que os produtos agrícolas fossem tratados da mesma maneira que outros produtos, especialmente porque a agricultura sempre foi considerada um setor "sensível" devido à sua grande contribuição para a segurança alimentar.

A segurança alimentar é levada em consideração em todas as partes do Acordo, começando com o Preâmbulo, depois com os artigos relacionados aos três pilares nos quais se baseia a regulamentação do comércio e concluindo com a norma dedicada à continuação do processo de reforma, que estava previsto na conclusão do Acordo. Sem entrar em detalhes sobre as regras relevantes, vale a pena observar como a segurança alimentar foi abordada, a fim de compreender o conceito de segurança alimentar emergente do sistema jurídico da OMC.

O parágrafo 6 do Preâmbulo do Acordo sobre Agricultura descreve que os Estados Membros observam "que os compromissos sob o programa de reforma devem ser feitos de forma equitativa entre todos os Membros, levando em conta preocupações não comerciais, incluindo a segurança alimentar e a necessidade de proteger o meio ambiente" (OMC, 1994). A questão da segurança alimentar é, portanto, levada em consideração e é caracterizada como uma preocupação não comercial à qual deve ser dada a devida consideração. A sua descrição como uma preocupação não comercial 
surgiu durante a "revisão intercalar" da rodada do Uruguai e enfraqueceu o modo como a questão da segurança alimentar era abordada no âmbito legal na OMC. A segurança alimentar tornou-se uma das possíveis preocupações a serem consideradas na elaboração de regras comerciais. Na verdade, os participantes das negociações reconheceram que outros fatores além da política comercial deveriam ser considerados na condução de suas políticas agrícolas. No mesmo sentido, o Artigo 20 do Acordo sobre Agricultura - que trata da continuação do processo de reforma a ser iniciado um ano antes do final do período de implementação (isto é, a partir de 2000) - ressalta que os Membros levarão em conta "preocupações não comerciais, tratamento especial e diferenciado para os PaísesMembros em desenvolvimento e o objetivo de estabelecer um sistema de comércio agrícola justo e orientado para o mercado"9 (OMC, 1994). A segurança alimentar é, portanto, incluída na agenda de negociações em curso (ou seja, a agenda de Doha para o Desenvolvimento), não como um capítulo autônomo da discussão, mas como uma das preocupações não comerciais.

Outros artigos do Acordo abordam explicitamente a segurança alimentar sob cada um dos seus três pilares. Em especial, no que se refere ao acesso ao mercado, o Anexo 5 introduz exceções à chamada "tarifação" - em certas condições - para a) produtos agrícolas primários e seus produtos trabalhados e/ou preparados e b) produtos agrícolas primários, que constituem o alimento predominante na dieta tradicional de um país Membro em desenvolvimento. Assim, em casos específicos que refletem fatores de preocupações não comerciais como a segurança alimentar, alguns tipos de produtos estritamente relacionados às necessidades nutricionais básicas estão sujeitos a tratamento especial. Em outras palavras, os Estados podem ser autorizados a manter medidas não tarifárias a fim de proteger sua produção agrícola da concorrência de produtos estrangeiros, a fim de garantir a segurança alimentar nacional.

No que diz respeito ao pilar de subsídios domésticos, a chamada "caixa verde" traz uma exceção às regras de redução de subsídios domésticos no caso de armazenagem pública para fins de segurança alimentar ${ }^{10}$ e ajuda alimentar ${ }^{11}$ em território nacional, entre muitos outros (OMC, 1994). Em outras palavras, essas regras comerciais permitem a compra (também a preços administrados para países em desenvolvimento ${ }^{12}$ ) de produtos agroalimentares com o objetivo de criar estoques nacionais de alimentos e para distribuí-los a pessoas em situação de vulnerabilidade.

Por fim, em relação às medidas relacionadas à exportação, é preciso mencionar o artigo 12 do Acordo, que é dedicado às proibições e restrições à exportação. De acordo com o pilar pertinente, os subsídios à exportação e outros métodos utilizados para tornar as exportações artificialmente competitivas estão sujeitos ao compromisso de redução (que, após a Conferência Ministerial de Nairóbi, tornou-se um compromisso de eliminação ${ }^{13}$ ). Além disso, as exportações não devem ser restringidas ou proibidas. Esta regra de proibição de exportação, de acordo com o artigo XI, alínea 2 (a) do GATT, encontra uma exceção no caso de escassez crítica de alimentos. Quando qualquer Membro da OMC aplicar essa exceção, deverão ser observadas as disposições previstas no artigo 12 do Acordo sobre Agricultura que, entre outras condições, exige que os Estados considerem os efeitos de tal proibição ou restrição à segurança alimentar do Membro importador (ANANIA, 2014). 


\section{O CONCEITO DE SEGURANÇA ALIMENTAR ORIUNDO DO SISTEMA JURÍ- DICO DA OMC: CONSIDERAÇÕES FINAIS}

A breve referência aos artigos explicitamente relacionados com a segurança alimentar ${ }^{14}$ mostra claramente a estrutura do conceito que parece ser coerente no âmbito do sistema legal da OMC. A segurança alimentar é concebida como uma exceção, uma razão válida para permitir exceções às regras gerais de comércio. Como as regras de comércio limitam os Estados, isso significa que as exceções estendem os poderes dos Estados no caso de questões de segurança alimentar. Essa conclusão confirma o reconhecimento da conexão entre segurança comercial e segurança alimentar pelos Estados membros da OMC.

Importante notar que a noção de segurança alimentar adotada considera os níveis nacional e global, e não os níveis individual e familiar. A ideia de segurança alimentar expressa no Acordo sobre Agricultura não acomoda a condição individual mas, em contrapartida, foca na autossuficiência agrícola de um país ou na adequação da autossuficiência alimentar nos níveis nacional e global ${ }^{15}$. Isso destaca o tipo de bifurcação no desenvolvimento do conceito de segurança alimentar dependendo do fórum considerado.

Por um lado, no âmbito das agências da ONU, emerge um conceito complexo e multidimensional, que toma forma ao final da Cúpula Mundial da Alimentação de 1996, organizada pela $\mathrm{FAO}^{16}$. Por outro lado, no mesmo período, o fórum do comércio internacional durante a Rodada Uruguai (1986-1994), que deu origem à OMC e ao Acordo sobre Agricultura, ainda contava com uma definição de segurança alimentar baseada na oferta. Foi mantida quase a mesma noção de segurança alimentar delineada na Conferência Mundial sobre Alimentação de 1974 (ONU, 1974) ("Disponibilidade em todos os momentos de suprimento adequado de alimentos básicos para sustentar uma expansão constante do consumo de alimentos e compensar as flutuações na produção e nos preços" ${ }^{17}$ ), concentrando-se na disponibilidade de alimentos em detrimento do acesso à alimentação, das necessidades nutricionais individuais e das preferências alimentares.

Poder-se-ia argumentar que uma noção de segurança alimentar a nível nacional era a única concebível durante a negociação do Uruguai, à medida que os Membros se concentravam nas regras do comércio internacional. Se isto estivesse relacionado com os interesses e o mandato dos Estados-Membros, o mesmo deveria ser verdade, considerando a atual rodada de negociações para a continuação do processo de reforma. No entanto, este não é o caso. O conceito de segurança alimentar emergente da atual rodada de negociações mostra um quadro diferente, que incorpora a dimensão individual e, como consequência, o direito à alimentação, ausente no debate multilateral na Rodada Uruguai. De fato, se o comércio é um instrumento para o desenvolvimento, "com vistas a elevar os padrões de vida"18, deve considerar as pessoas e suas necessidades básicas.

\section{NOTAS}

1 Professora do Institute of Law, Politics and Development (Dirpolis) of the Sant'Anna School, Pisa/Itália. E-mail: mariagrazia.alabrese@santannapisa.it

2 O Relatório foi preparado coletivamente por 12 instituições globais e regionais líderes sob a égide da Rede de Informação de Segurança Alimentar (Food Security Information Network, FSIN). O FSIN é uma inicia- 
tiva global co-patrocinada pela FAO, WFP e IFPRI para fortalecer os sistemas de informação de segurança alimentar e nutricional. É financiado pela União Europeia e pela US Aid.

3 Esta definição representa uma elaboração posterior à mais citada, emitida no final da Cúpula Mundial da Alimentação de 1996, organizada pela FAO. A definição é apresentada no parágrafo 1 do Plano de Ação da Cúpula Mundial da Alimentação (desdobramento da Cúpula, juntamente com a Declaração de Roma sobre Segurança Alimentar Mundial) in verbis: "A segurança alimentar existe quando todas as pessoas, em todos os momentos, têm acesso físico e econômico a alimentos suficientes, seguros e nutritivos para atender às suas necessidades alimentares e preferências alimentares para uma vida ativa e saudável" (tradução nossa, do original: "Food security exists when all people, at all times, have physical and economic access to sufficient, safe and nutritious food to meet their dietary needs and food preferences for an active and healthy life") (FAO, 2009).

4 Uma descrição dos quatro pilares pode ser encontrada em SIMON, 2009, p. 19.

5 Em relação ao tópico da segurança alimentar antes da conclusão do Acordo sobre Agricultura no sistema multilateral de comércio, consultar MARGULIS, 2017, p. 25.

6 Tradução nossa, do original: "food security is not a matter for the rules per se, but is something separate [...]" (SMITH, 2012, p. 57.

7 Tradução nossa, do original: "that commitments under the reform programme should be made in an equitable way among all Members, having regard to non-trade concerns, including food security and the need to protect the environment" (OMC, 1994).

8 Ver Summary of main points raised at the twelfth meeting of the negotiating group on agriculture - Note by the Secretariat, MTN.GNG/NG5/W/93, 13 January 1989, parágrafo 28; e em especial o document Midterm Meeting, MTN.TNC/11, 21 April 1989, p. 10-11.

9 Tradução nossa, do original: "non-trade concerns, special and differential treatment to developing country Members, and the objective to establish a fair and market-oriented agricultural trading system" (OMC, 1994).

10 Ver o Anexo 2 do Acordo sobre Agricultura, parágrafo 3 (OMC, 1994).

11 Ver o Anexo 2 do Acordo sobre Agricultura, parágrafo 4 (OMC, 1994).

12 Ver o Anexo 2 do Acordo sobre Agricultura, parágrafo 3, nota 5 (OMC, 1994) que torna claro que, no caso de preços administrados, a diferença entre o preço de aquisição e o preço de referência externo é contabilizada no AMS (sujeito a redução). Sobre este tema vale mencionar a cláusula de paz introduzida pela chamada decisão de Bali (Ministerial Decision, Public stockholding for food security purposes, 11th December 2013, WT/MIN(13)/38), confirmada pela decisão do Conselho Geral WT/L/939. A decisão subsequente, decisão de Nairobi WT/MIN(15)/44, December 2015, não mudou o quadro.

13 Ver Ministerial Decision, Export Competition, 19th December 2015, WT/MIN(15)/45.

14 O Artigo 10.4 do Acordo sobre Agricultura não foi mencionado pois não inclui uma referência explícita à segurança alimentar, embora sua relevância para o tópico seja inegável.

15 "A segurança alimentar é tradicionalmente discutida em termos de autossuficiência alimentar em dois sentidos. O primeiro requer a produção de alimentos nas quantidades consumidas domesticamente, enquanto o segundo requer disponibilidade doméstica. A autossuficiência em sua primeira acepção exclui as importações como uma importante fonte de abastecimento, enquanto a segunda não apresenta essa restrição" (do original: "Food security is traditionally discussed in terms of either food self-sufficiency or food self-reliance. The former requires production of food in the quantities consumed domestically, while the latter requires domestic availability. Self-sufficiency rules out imports as a major source of supply while self-reliance has no such restriction") (FAO, 2003, p. 35). 


\section{Ver nota 2.}

17 Tradução nossa, do original: "Availability at all times of adequate world food supplies of basic foodstuffs to sustain a steady expansion of food consumption and to offset fluctuations in production and prices" (ONU, 1974).

18 Conforme o preâmbulo do Acordo que estabeleceu a Organização Mundial do Comércio.

\section{REFERÊNCIAS}

ANANIA, G. Export Restrictions and Food Security. In: R. MELÉNDEZ-ORTIZ, R.; BELLMANN, C.; HEPBURN, J. (Editors). Tackling Agriculture in the Post-Bali Context: A collection of short essays. International Centre for Trade and Sustainable Development, Switzerland, 2014, pp. 183-195.

FAO, IFAD, UNICEF, WFP, WHO. The State of Food Security and Nutrition in the World 2017. Building resilience for peace and food security. FAO, 2017. Disponível em: http://www.fao.org/3/a-I7695e.pdf. Acesso em: 30 jan. 2019.

FOOD AND AGRICULTURE ORGANIZATION (FAO). Trade reforms and food security. Conceptualizing the linkages, 2003.

FOOD AND AGRICULTURE ORGANIZATION (FAO). Declaration of the World Summit on Food Security. 2009. Disponível em: http://www.fao.org/fileadmin/templates/wsfs/Summit/Docs/Declaration/ WSFS09_Draft_Declaration.pdf. Acesso em: 30 jan. 2019.

FOOD SECURITY INFORMATION NETWORK (FSIN). Global Report on Food Crises. 2018. Disponível em: http://www.fsincop.net/resource-centre/detail/en/c/1110426/. Acesso em: 30 jan. 2019.

MATTHEWS, A. Trade Rules, Food Security and the Multilateral Trade Negotiations. In: European Review of Agricultural Economics, v. 21, n. 3, 2014, pp. 511-535.

CLAPP, J. Food security and international trade: Unpacking disputed narratives. FAO, Rome, 2015.

MARGULIS, M. E. The Forgotten History of Food Security in Multilateral Trade Negotiations. In: World Trade Review, v. 16, n.1, 2017, pp. 25-57.

SIMON, G. A. Concepto y gobernanza internacional de la seguridad alimentaria: de donde venimos y hacia donde vamos. In: Revista Española de Estudios Agrosociales y Pesqueros, n. 224, 2009, pp. 19-35.

Organização das Nações Unidas (ONU). Report of the World Food Conference. Rome, November 1974. Disponível em: https://digitallibrary.un.org/record/701143/files/E_CONF.65_20-EN.pdf. Acesso em: 30 jan. 2019.

ONU, General Assembly. Transforming our world: the 2030 Agenda for Sustainable Development, adopted by the UN General Assembly Resolution, 25 September 2015, A/RES/70/1.

ONU, General Assembly. United Nations Decade of Action on Nutrition (2016 - 2025). Adopted by the UN General Assembly Resolution, 1 April 2016, A/RES/70/259.

SMITH, F. Food security and International agricultural trade regulation: old problems, new perspectives. In: Research Handbook on the WTO Agriculture Agreement: New and Emerging Issues in International Agricultural Trade Law, MCMAHON, J. A.; DESTA, M. G. (Editors). Edward Elgar Publishing, Cheltenham, UK-Northampton, MA, USA, 2012.

Organização Mundial do Comércio (OMC). Summary of main points raised at the twelfth meeting of the negotiating group on agriculture - Note by the Secretariat, MTN.GNG/NG5/W/93, 13 January 1989.

Organização Mundial do Comércio (OMC). Agreement on Agriculture, 1994. Disponível em: https://www. wto.org/english/docs_e/legal_e/14-ag_02_e.htm. Acesso em: 30 jan. 2019.

Organização Mundial do Comércio (OMC).. Mid-term Meeting, MTN.TNC/11, 21 April 1989, p. 10-11. 
Os contornos da segurança alimentar no âmbito do sistema jurídico multilateral de comércio ALABRESE, $M$.

Data de submissão do artigo: Agosto de 2019

Data de aceite do artigo: Novembro de 2019 